\title{
Distribution Characteristics of Drug Susceptibility Test Results of Tuberculosis and Non-Tuberculous Bacilli in Patients with Opportunistic Infections of AIDS
}

\author{
Qing Lin ${ }^{*}$, Lida Mo ${ }^{2,3}{ }^{*}$, Xiaoye Su ${ }^{4,5}$, Lihua Qin ${ }^{4,6,7 \#, ~ G u o s h e n g ~ S u} u^{8,9 \#}$ \\ ${ }^{1}$ Department of Infectious Diseases, People's Hospital of Debao County, Debao, China \\ ${ }^{2}$ Department of Medical Laboratory Science, Nanning Fourth People's Hospital, Nanning Infectious Disease Hospital Affiliated to \\ Guangxi Medical University, Nanning, China \\ ${ }^{3}$ Guangxi AIDS Clinical Treatment Center, Nanning, China \\ ${ }^{4}$ Department of Nursing, Graduate College of Eternity, University of the Philippines, Quezon City, Philippines \\ ${ }^{5}$ Department of Critical Care Medicine, Fuzhou Second People's Hospital, Fuzhou, China \\ ${ }^{6}$ The People's Hospital of the Guangxi-ASEAN Economic and Technological Development Zone, Nanning, China \\ ${ }^{7}$ The First Department of Internal Medicine, Nanning Tenth People's Hospital, Nanning, China \\ ${ }^{8}$ Department of Laboratory Medicine, West China Hospital, Sichuan University, Chengdu, China \\ ${ }^{9}$ Department of Laboratory Medicine, The People's Hospital of the Guangxi-ASEAN Economic and Technological Development \\ Zone, Nanning Tenth People's Hospital, Nanning, China \\ Email:"563449581@qq.com
}

How to cite this paper: Lin, Q., Mo, L.D., $\mathrm{Su}$, X.Y., Qin, L.H. and Su, G.S. (2021) Distribution Characteristics of Drug Susceptibility Test Results of Tuberculosis and Non-Tuberculous Bacilli in Patients with Opportunistic Infections of AIDS. Journal of Tuberculosis Research, 9, 256-265.

https://doi.org/10.4236/jtr.2021.94024

Received: September 22, 2021

Accepted: November 7, 2021

Published: November 10, 2021

Copyright $\odot 2021$ by author(s) and Scientific Research Publishing Inc. This work is licensed under the Creative Commons Attribution International License (CC BY 4.0).

http://creativecommons.org/licenses/by/4.0/ (c) (i) Open Access

\begin{abstract}
Objective: To understand the distribution of drug susceptibility test results of opportunistic infections of tuberculosis and non-tuberculous bacilli in AIDS patients. Methods: The AIDS patients who were hospitalized in our hospital from January 2016 to June 2019 were collected as the research objects, and patients with opportunistic tuberculosis and non-tuberculous bacilli from AIDS patients were screened for drug susceptibility tests, and the distribution characteristics of drug susceptibility were analyzed. Results: 179 strains of tuberculosis and non-tuberculous mycobacteria were isolated from the specimens of AIDS patients, including 135 cases of tuberculosis mycobacteria and 44 cases of non-tuberculous mycobacteria. In the results of the drug susceptibility test, most strains of Mycobacterium tuberculosis showed sensitivity to commonly used drugs, and a few strains showed resistance; most strains of non-tuberculous mycobacteria showed resistance, and a few strains showed sensitivity. Conclusion: AIDS opportunistic infection of Mycobacterium
\end{abstract}


tuberculosis and non-tuberculous mycobacteria have significant differences in drug sensitivity test results. Timely detection and analysis are of great significance to the diagnosis and treatment of the disease.

\section{Keywords}

AIDS, Opportunistic Infections, Mycobacterium Tuberculosis Complex, Non-Tuberculous Mycobacteria

\section{Introduction}

AIDS opportunistic infection is a secondary pathogen infection that occurs because people are infected with AIDS virus, which leads to low immunity of the body [1]. For people with normal immunity, these pathogen infections rarely occur. However, AIDS patients have reduced immunity and are prone to opportunistic infections with these pathogens. Once infected, they will aggravate the disease and cause various complications and endanger life, even death [2] [3]. Therefore, when HIV infection is diagnosed, it is necessary to assess the condition in time to determine whether there is opportunistic pathogen infection [4]. If there is indeed a relevant opportunistic infection, it is necessary to formulate a corresponding treatment plan in time.

At present, the main pathogens of secondary infections are Mycobacterium tuberculosis infection, non-tuberculous mycobacterium infection, Pneumocystis pneumonia, fungal infection, cytomegalovirus infection, toxoplasma infection, herpes simplex virus and varicella-zoster virus infection, etc. [5]. Among them, Mycobacterium tuberculosis infection and non-tuberculous mycobacterium infection are very common opportunistic infections [6]. In order to understand the distribution of drug susceptibility test results for AIDS opportunistic infections of tuberculosis and non-tuberculous mycobacteria, this study collected 3 years of data for analysis. The results are now reported as follows.

\section{Materials and Methods}

\subsection{Listed as Research Objects}

The 179 cases of AIDS opportunistic infections with tuberculosis and non-tuberculous mycobacteria were selected from patients who were hospitalized in the Infectious Diseases Department of the Fourth People's Hospital of Nanning from January 2016 to June 2019. Among them, there were 135 cases of Mycobacterium tuberculosis infection and 44 cases of non-tuberculous mycobacteria infection. Comparison of the number of tuberculosis and non-tuberculous infection cases, $\chi^{2}=92.5251, \mathrm{P}=0.0000$, all study subjects were excluded from other pathogen infections. Among the 179 patients, 151 were males, aged 23 - 86 years old, with an average age of $(49.79 \pm 14.42)$ years old; 28 female patients were aged between 14 - 90 years old, with an average age (43.57 \pm 17.28$)$ years old, 
Comparison of the number of male and female infection cases, $\chi^{2}=169.0391, \mathrm{P}$ $=0.0000$; comparison of the average age of male and female infection, $t=2.0299$, $\mathrm{P}=0.0251$. All study subjects were signed with the patient's own consent and approved by the Medical Ethics Committee for the study.

\subsection{Research Methods}

AIDS patients hospitalized in the Fourth People's Hospital of Nanning from January 2016 to June 2019 were selected. Patients with suspected opportunistic infection or symptoms of opportunistic infection were selected for corresponding specimens and culture medium for ordinary bacteria culture, specimens with common pathogenic bacteria growth for bacterial identification and drug sensitivity test, and drug susceptibility test for tuberculosis and non-tuberculosis identification and drug sensitivity test.

\subsection{Apparatus and Reagents}

Common bacteria identification and drug susceptibility equipment adopts the integrated identification and drug susceptibility equipment produced by Hunan Tiandiren Company and French Mérieux Company; Identification and drug sensitivity test of $M$. tuberculosis were performed by mycobacterium drug susceptibility detection kit (culture method). Drug sensitivity plates and culture tubes are shown below in Figures 1-3. The reagents use the supporting reagents produced by the equipment manufacturer, and all reagents are used within the validity period.

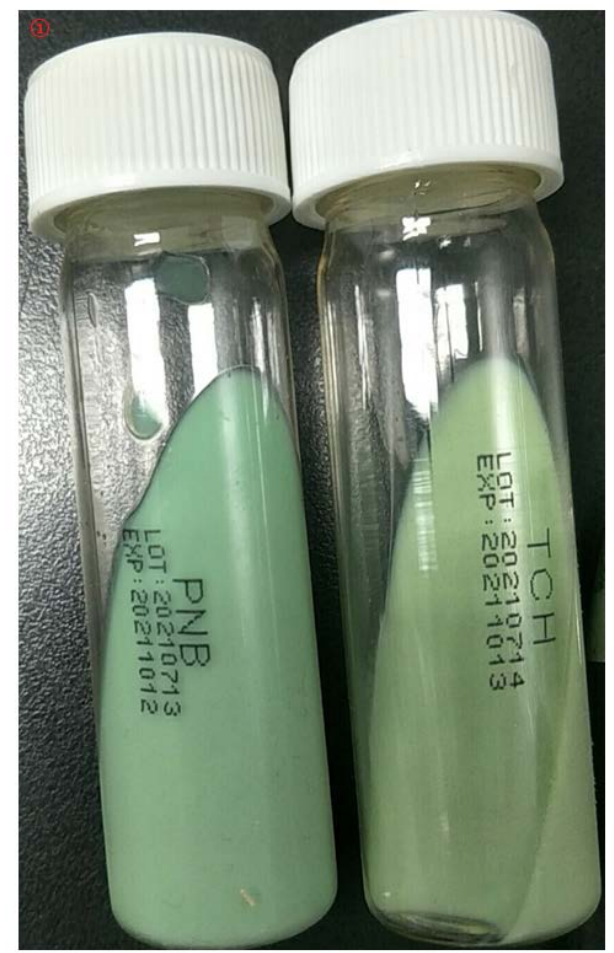

Figure 1. Mycobacterium culture medium. 


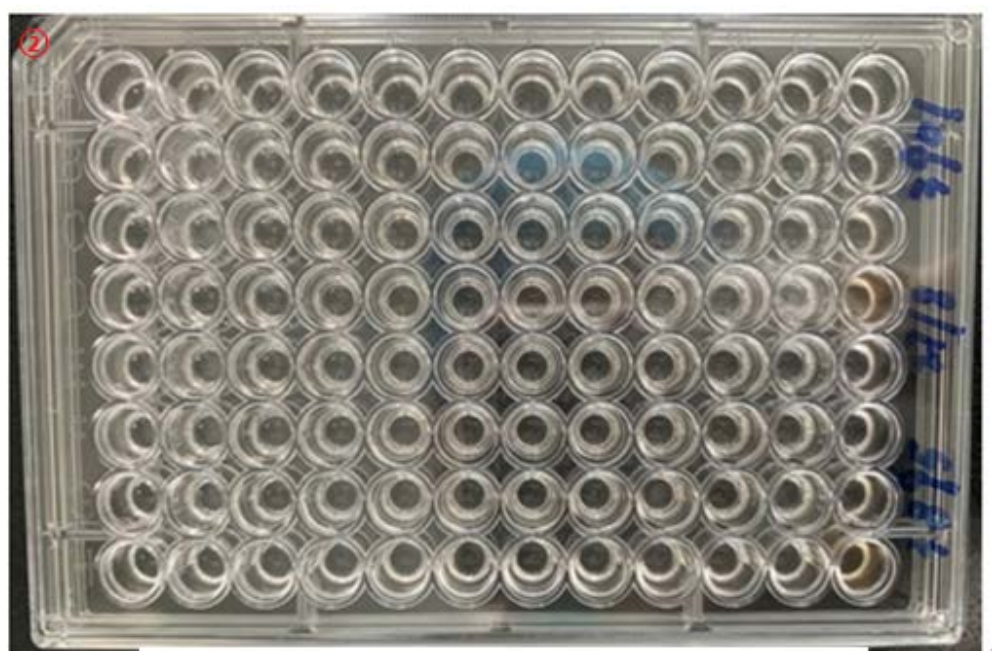

Figure 2. Drug sensitivity test of Mycobacterium tuberculosis complex group.

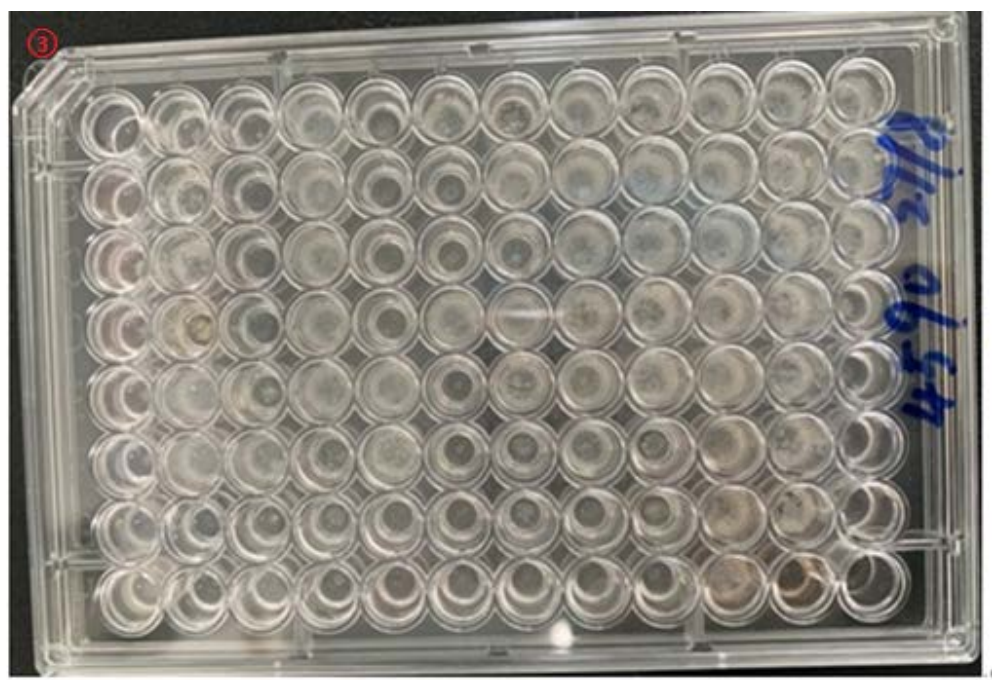

Figure 3. Drug susceptibility test for non-tuberculous mycobacteria.

\subsection{Results of Tuberculosis and Non-Tuberculous Mycobacteria}

1) Interpretation of the culture result: Observe the result after 7 days, the bottom of the well is positive for white bacterial deposits, negative for sterile deposits, and negative holes. Observe the result again after 10 days. If white bacterial deposits appear, it is positive, and there is still nothing. The bacterial precipitation is negative (note: when it is difficult to determine the MIC well, the well can be compared with $1 / 10$ of the reference well, if the bacterial precipitation in the well is less than $1 / 10$ of the reference well, the growth can be judged to be inhibited); 2) The criteria for strain determination are shown in Table 1;3) MIC interpretation: when the positive control wells (A2, B2 or E2, F2) are positive, the result will be interpreted, and the MIC value of each drug will be recorded. The MIC value is the lowest of the colorless white precipitate in the drug concentration hole, record the MIC value on the result recording paper. The drug distribution diagram of the microplate is shown in Table 2 below. 
Table 1. Judgment criteria for Mycobacterium tuberculosis and non-tuberculous mycobacteria.

\begin{tabular}{cccc}
\hline Strain name & Control & TCH & PNB \\
\hline Mycobacterium tuberculosis of the human type & + & + & - \\
Mycobacterium tuberculosis of the bovine type & + & - & - \\
NTM & + & + & + \\
\hline
\end{tabular}

Table 2. Schematic representation of the MIC micropore distribution.

\begin{tabular}{|c|c|c|c|c|c|c|c|c|c|c|c|c|c|}
\hline & & 1 & 2 & 3 & 4 & 5 & 6 & 7 & 8 & 9 & 10 & 11 & 12 \\
\hline \multirow{2}{*}{ A } & \multirow{2}{*}{$\mathrm{E}$} & \multirow{2}{*}{ 1/10Ref } & \multirow{2}{*}{ GC } & S & I & $\mathrm{R}$ & $\mathrm{E}$ & $\mathrm{Rft}$ & Lfx & $\mathrm{Am}$ & $\mathrm{Cm}$ & Pto & PI \\
\hline & & & & 1 & 0.2 & 1 & 2.5 & 0.5 & 2 & 1 & 2.5 & 10 & 0.5 \\
\hline B & $\mathrm{F}$ & 1/10Ref & GC & 2 & 0.4 & 2 & 5 & 2 & 8 & 4 & 10 & 40 & 2 \\
\hline \multirow{2}{*}{$\mathrm{C}$} & \multirow{2}{*}{ G } & \multirow{2}{*}{$\mathrm{NC}$} & $\mathrm{TCH}$ & \multirow{2}{*}{4} & \multirow{2}{*}{0.8} & \multirow{2}{*}{4} & \multirow{2}{*}{10} & Mfx & Pas & Ctr & $\mathrm{Rfb}$ & KAN & $\mathrm{Cfz}$ \\
\hline & & & 0.25 & & & & & 0.5 & 2 & 4 & 0.75 & 2.5 & 2 \\
\hline $\mathrm{D}$ & $\mathrm{H}$ & $\mathrm{NC}$ & $\begin{array}{r}\text { PNB } \\
400\end{array}$ & 8 & 1.6 & 8 & 20 & 2 & 8 & 16 & 3 & 10 & 8 \\
\hline
\end{tabular}

\subsection{Statistical Analysis}

The SPSS22.0 statistical software was used for analysis. The chi-square test was used for the comparison of the number of related infections; the $t$ test was used for the comparison of related values, and $\mathrm{P}<0.05$ was considered statistically significant.

\section{Results}

179 strains of tuberculosis and non-tuberculous mycobacteria were isolated from the collected specimens of AIDS patients. Among them, 135 cases of Mycobacterium tuberculosis, accounting for $75.42 \%$ of mycobacterial infections, and 44 cases of non-tuberculous mycobacteria, accounting for mycobacterial infections $24.58 \%$, comparing the number of tuberculosis and non-tuberculous infection cases, $\chi^{2}=92.5251, \mathrm{P}=0.0000$, all study subjects were excluded from infection with other pathogens, see Table 3 for details. Among the 179 patients, 151 were males, accounting for $84.36 \%$, and they were 23 - 86 years old, with an average age of ( $49.79 \pm 14.42)$ years; 28 females, accounting for $15.64 \%$, were between 14 - 90 years old, and the average age was $(43.57 \pm 17.28)$ years old; comparison of the number of infection cases between men and women, $\chi^{2}=169.0391, \mathrm{P}=$ 0.0000; comparison of the average age of infection between men and women, $\mathrm{t}=$ $2.0299, \mathrm{P}=0.0251$, see Table 4 for details. In the results of the drug susceptibility test, tuberculosis and non-tuberculous mycobacteria have a large gap in susceptibility to the nine commonly used antibiotics, butylamkanamycin, amino salicylate, curamycin, rifampicin, streptomycin, ethambubutol, isoniazid, levoxofloxacin. In addition to the sensitivity of nontuberculous mycobacteria to isonia- 
zid up to $84.09 \%$, the sensitivity to other antibiotics is not more than $35 \%$, and the highest drug resistance rate is $97.73 \%$. The sensitivity of the Mycobacterium tuberculosis complex to 9 commonly used antibiotics is more than $77.78 \%$, and the highest sensitivity to prothionamide can reach $100 \%$. The drug susceptibility of tuberculosis and non-tuberculous mycobacteria to nine commonly used antibiotics and the comparison of tuberculosis and non-tuberculous mycobacteria to various drugs are shown in Table 5 below.

Table 3. Comparison of the distribution of 179 cases of tuberculosis and non-tuberculous mycobacteria.

\begin{tabular}{cc}
\hline Strain distribution & Number of cases (\%) \\
\hline Mycobacterium tuberculosis complex & $135(75.42)$ \\
Non-tuberculous mycobacteria & $44(24.58)$ \\
$\chi^{2}$ value & 92.5251 \\
P value & 0.0000 \\
\hline
\end{tabular}

Table 4. Comparison of sex and age distribution of 179 cases of tuberculosis and nontuberculous mycobacteria.

\begin{tabular}{ccc}
\hline Gender & Number of cases (\%) & Average age (years) \\
\hline Male & $151(84.36)$ & $49.79 \pm 14.42$ \\
Female & $28(15.64)$ & $43.57 \pm 17.28$ \\
$\chi^{2}$ or t value & 169.0391 & 2.0299 \\
P value & 0.0000 & 0.0000 \\
\hline
\end{tabular}

Table 5. Comparison of susceptibility test results of tuberculosis and non-tuberculous mycobacteria to commonly used drugs.

\begin{tabular}{ccccccc}
\hline \multirow{2}{*}{ Drug name } & $\begin{array}{c}\text { Non-tuberculous } \\
\text { mycobacteria }\end{array}$ & \multicolumn{2}{c}{$\begin{array}{c}\text { Mycobacterium } \\
\text { tuberculosis complex }\end{array}$} & $\chi^{2}$ value & P value \\
\cline { 2 - 5 } & $\mathrm{S}(\mathrm{n})$ & $\mathrm{R}(\mathrm{n})$ & $\mathrm{S}(\mathrm{n})$ & $\mathrm{R}(\mathrm{n})$ & & \\
\hline Prothionamide & 37 & 7 & 135 & 0 & 18.3181 & 0.0000 \\
Amikacin & 13 & 31 & 130 & 5 & 92.0268 & 0.0000 \\
Aminosalicylic acid & 1 & 43 & 125 & 10 & 129.8852 & 0.0000 \\
Capreomycin & 11 & 33 & 132 & 3 & 109.3953 & 0.0000 \\
Rifampicin; & 1 & 43 & 118 & 17 & 107.9330 & 0.0000 \\
Streptomycin & 6 & 38 & 112 & 23 & 70.9949 & 0.0000 \\
Ethambutol & 13 & 31 & 128 & 7 & 84.5387 & 0.0000 \\
Isonicid & 1 & 43 & 105 & 30 & 78.3362 & 0.0000 \\
Levofloxacin & 15 & 29 & 122 & 13 & 58.5288 & 0.0000 \\
\hline
\end{tabular}




\section{Discussion}

Mycobacterium tuberculosis, commonly known as tuberculosis, causes human tuberculosis, and the main pathogenic bacterium is human Mycobacterium tuberculosis ( $M$. tuberculosis), M. bovine tuberculosis ( $M$. bovis), generally referring to M. human tuberculosis and M. bovine M. tuberculosis infection collectively as the M. tuberculosis complex group. According to statistics, one-third of people in the world are infected with Mycobacterium tuberculosis, and about $70 \%$ of Mycobacterium tuberculosis infections occur in Asia; Due to the high infection rate of Mycobacterium tuberculosis, the World Health Organization has designated March 24 every year as World Tuberculosis Day [6]. Non-tuberculous mycobacteria refer to mycobacteria other than human Mycobacterium tuberculosis, Mycobacterium bovis and Mycobacterium leprosy [7]. It is relatively sensitive to acid and base, has strong tolerance to commonly used anti-tuberculosis drugs, and has less strict growth environment as required as $\mathrm{My}$ cobacterium tuberculosis. It exists in various environments and is mostly conditional pathogenic bacteria, which has attracted much attention because it can cause human tuberculosis-like lesions. AIDS has a higher chance of opportunistic infection of Mycobacterium tuberculosis complex and non-tuberculous mycobacteria. It is a common complication of AIDS patients. Once co-infection, especially non-tuberculous mycobacterial infection, can cause disease aggravation; So timely inspection and timely treatment are needed to relieve the condition in time and improve the survival rate and quality of life of AIDS patients [8] [9].

The results of this study show that in the collection of three and a half years of hospitalized AIDS patients, 179 cases of tuberculosis and non-tuberculous mycobacterial infections were isolated, and 135 cases of Mycobacterium tuberculosis complex infections were isolated, occupying a larger part of non-tuberculous mycobacteria. While, 44 cases of mycobacterial infection, also occupy a lot of part. This shows that in patients with AIDS opportunistic mycobacteria, the proportion of Mycobacterium tuberculosis complex infection is relatively high, and the proportion of non-tuberculous mycobacteria infection is relatively low [10] [11]. Among the male and female infection ratios, the male infection rate is relatively high, reaching 151 cases, and the female infection rate is relatively low, only 28 cases. This shows that among the patients with AIDS opportunistic infections studied, it is possible that the proportion of male AIDS patients is relatively high, resulting in an unbalanced ratio of male and female infections [12] [13]; In addition, the ratio of men to women in China is inherently unbalanced. The most obvious feature of the ratio of men to women is that there are more men than women. Taking 1.4 billion people as an example, there are 715.27 million men and 684.7 million women. This data can clearly see that the ratio of men to women in China is not balanced, with nearly 30 million more men than women. Therefore, this study found that the uneven number of male and female infections is a normal result.

The results of this study also showed that there was a large gap between the 
susceptibility of tuberculosis and non-Mycobacterium tuberculosis to nine commonly used antibiotics. Among the nine commonly used antibiotics, the $\mathrm{Myco}$ bacterium tuberculosis complex group showed high sensitivity to various commonly used antibiotics, with the lowest sensitivity of $77.78 \%$ and the highest sensitivity of $100 \%$ [14]; However, non-Mycobacterium tuberculosis showed high sensitivity to thioisoniotinamide, reached $84.09 \%$ and showed high resistance to other drugs, with a maximum rate of $97.73 \%$; This shows that Mycobacterium tuberculosis has high sensitivity to various commonly used antibiotics [15] [16]; non-tuberculous mycobacteria have strong resistance to various commonly used antibiotics, which has a great challenge for the treatment of opportunistic AIDS infection with non-tuberculous mycobacteria [17].

\section{Conclusion}

Opportunistic infections of AIDS with tuberculosis and non-tuberculous mycobacteria are common complications of AIDS. Mycobacterium tuberculosis and non-tuberculous mycobacteria have big differences in drug sensitivity to commonly used antibiotics. Timely screening and timely symptomatic treatment are the only diagnosis and treatment plan for AIDS patients, which is conducive to improving the survival time and quality life of AIDS patients.

\section{Limitations of the Study}

This study mainly analyzes the distribution characteristics of drug susceptibility to AIDS opportunistic infections of tuberculosis and non-tuberculous mycobacteria in this region. Since all cases are from this region, there are certain differences in drug sensitivity, which cannot fully represent the situation in other regions. Therefore, it has certain limitations.

\section{Acknowledgements}

This project has been approved, with strong support from the functional departments of the hospital and the help of my colleagues. I hereby express my heartfelt thanks for their support and help! I wish them good health, smooth work and all the best!

\section{Fund Project}

Scientific Research Project of Health and Family Planning Commission of Guangxi Zhuang Autonomous Region (NO.: Z2016067).

\section{Conflicts of Interest}

The authors declare no conflicts of interest regarding the publication of this paper.

\section{References}

[1] AIDS and Hepatitis C Group of Infectious Diseases Branch of Chinese Medical As- 
sociation, Chinese Center for Disease Control and Prevention (2018) Guidelines for Diagnosis and Treatment of AIDS in China (2018 Edition). China AIDS and STDS, 24, 1266-1282.

[2] Wu, Z. (2018) The New Characteristics of Sexual Transmission of AIDS in My Country and the Challenges of Prevention and Treatment. Chinese Journal of Epidemiology, 39, 707-709.

[3] Naseer, M., Dailey, F.E., Juboori, A.A., Samiullah, S. and Tahan, V. (2018) Epidemiology, Determinants, and Management of AIDS Cholangiopathy: A Review. World Journal of Gastroenterology, 24, 767-774. https://doi.org/10.3748/wjg.v24.i7.767

[4] Xiao, G., Xiao, Y., Chen, X., et al. (2019) Clinicopathological Characteristics of AIDSRelated Opportunistic Infectious Diseases. Journal of Guangzhou Medical University, 47, 22-28.

[5] Sun, Y., Chen, Z., Zhao, Q., et al. (2018) Comparative Analysis of AIDS Patients with Opportunistic Infections in Different Periods from 2009 to 2016 in Henan Province. Chinese Journal of Infectious Diseases, 36, 678-680.

[6] Ren, T., Deng, D., Fu, L., et al. (2020) 2020 WHO Global Tuberculosis Report: Analysis of Key Data from Global and China. E-Journal of Emerging Infectious Diseases, $5,280-284$.

[7] Wu, Y., Deng, X., Hu, F., et al. (2017) Pathogen Spectrum, Clinical Features and Drug Resistance of AIDS Combined with Nontuberculous Mycobacterial Lung Disease. Chinese Journal of Infectious Diseases, 35, 142-145

[8] Ye, W., Wu, S., Chen, Y., et al. (2020) Clinical Characteristics of Opportunistic Infections in HIV/AIDS Patients with Different Baseline CD4+ T Cell Levels in Antiretroviral Therapy. Chinese Journal of Nosocomial Infection, 30, 2296-2300.

[9] Xue, S., Wu, G., He, W., et al. (2017) Observation on the Effect of Anti-Tuberculosis Drug Treatment in Patients with AIDS Complicated with Tuberculosis. Journal of Tuberculosis and Lung Health, 6, 32-35.

[10] Liu, L., Zhang, R., Tang, Y., Qi, T., Song, W., Wang, Z., et al. (2018) The Importance of Nontuberculous mycobacteria Identification in Chinese Patients Infected with HIV. BioScience Trends, 12, 515-516. https://doi.org/10.5582/bst.2018.01254

[11] Xu, Y., Li, Q., Zhu, M., et al. (2019) Analysis of Characteristics and Drug Resistance of Mycobacterial Infection in HIV-Infected Patients in Sichuan. Chinese Journal of Nosocomial Infection, 29, 34-38.

[12] Meng, S., Wu, F., Chen, X., et al. (2017) Analysis of Dual Infection of AIDS and Tuberculosis in Guangxi and Its Influencing Factors. Chinese Journal of New Clinical Medicine, 10, 299-302.

[13] Liang, P., Shen, Y., Liu, L., et al. (2017) Clinical Epidemiological Study on 359 Cases of Acquired Immunodeficiency Syndrome Complicated with Pulmonary Tuberculosis. Chinese Journal of Infection and Chemotherapy, 17, 233-237

[14] Wang, Y., Zhu, Y., Zhou, R., et al. (2017) Drug Resistance Analysis of 196 Strains of Mycobacterium tuberculosis in HIV/AIDS Patients with Tuberculosis. Chongqing Medicine, 46, 1203-1205.

[15] Chen, Y., Liu, X., Wang, Y., et al. (2021) Analysis of Initial Drug Resistance of $M y$ cobacterium tuberculosis in HIV/TB Co-Infected Patients in Henan. China AIDS and STDs, 27, 14-16.

[16] Cao, J., Cai, X., Yao, J., et al. (2018) Drug Resistance of Mycobacterium tuberculosis in Patients with Acquired Immunodeficiency Syndrome Complicated with Pulmonary Tuberculosis. Chinese Journal of Clinical Pharmacy, 27, 260-263. 
[17] Deng, X., Chen, W., Lan, Y., et al. (2015) Analysis of Drug Resistance in HIV/AIDS Patients Co-Infected with Nontuberculous mycobacteria in Guangzhou. Journal of Tropical Medicine, 15, 170-173. 